\title{
Efeitos do estímulo acústico rítmico na marcha de pacientes com Doença de Parkinson
}

\author{
Effect of rhythmic auditory cue on gait in patients with Parkinson's Disease \\ Luize Matsumoto' ${ }^{1}$ Glaucia Magalhães², Gisele Ladik Antunes', \\ Camila Torriani-Pasin ${ }^{4}$
}

\section{RESUMO}

Objetivo. Investigar quais parâmetros da marcha sofrem maior alteração com Estímulo Acústico Rítmico (EAR) em pacientes com Doença de Parkinson (DP). Método. Foram avaliados 21 sujeitos diagnosticados com DP, com média de idade de $69 \pm 11,6$, por meio do teste de caminhada dos 10 metros em duas situaçôes: em marcha livre e com auxílio de EAR, calculado em $10 \%$ acima da marcha preferida. As variáveis dependentes foram cadência, comprimento de passo e velocidade de marcha e foram comparadas através do coeficiente de variação. $\mathrm{O}$ Teste $\mathrm{t}$ para as variáveis dependentes foi utilizado, considerando-se o nível de significância de 5\% para todas as análises. Resultados. Houve diferença entre marcha livre e marcha com EAR para as variáveis: velocidade $(\mathrm{p}<0,001)$, cadência $(\mathrm{p}<0,001)$ e comprimento de passo $(\mathrm{p}=0,015)$, com valores mais altos para a marcha com EAR em comparaçáo à marcha livre. $\mathrm{O}$ cálculo de coeficiente de variação apresentou maior variação no parâmetro velocidade $(9,6 \%)$, comparado à cadência $(5,3 \%)$ e comprimento de passo $(4,3 \%)$. Conclusáo. $\mathrm{O}$ uso de estímulo acústico rítmico em $10 \%$ acima da frequência preferida aumenta a velocidade em comparaçáo à cadência e comprimento de passo de indivíduos com DP.

Unitermos. Parkinson, Estímulo Auditivo, Marcha

Citação. Matsumoto L, Magalhães G, Antunes GL, Torriani-Pasin C. Efeitos do estímulo acústico rítmico na marcha de pacientes com Doença de Parkinson.

\begin{abstract}
Objective. To determine which gait parameters is more affected by the use of Rhythmic Auditory Cue (RAC) in patients with Parkinson's Disease. Method. 21 patients with a diagnosis of PD were evaluated, with a mean age of $69 \pm 11.6$, by a 10 meter gait test at two situations: unaided walking and with the aid of RAC calculated at $10 \%$ above the preferred gait. Cadence, stride length and gait speed were compared using the coefficient of variation. Test $\mathrm{T}$ for dependent variables was used considering a significance level of $5 \%$ for all analyzes. Results. We found a difference between unaided walking and walking with RAC for the variables: gait speed $(\mathrm{p}<0.001)$, cadence $(\mathrm{p}<0.001)$ and stride length $(\mathrm{p}=0.015)$, with higher values for walking with $\mathrm{RAC}$ compared to unaided walking. The calculation of the coefficient of variation showed greater variation in the speed $(9.6 \%)$ parameter compared to cadence $(5.3 \%)$ and step length (4.3\%). Conclusion. the use of RAC in $10 \%$ above the preferred gait leads to an increase in gait parameters with the greatest change in speed gait in comparison to the cadence and stride length in people with PD.
\end{abstract}

Keywords. Parkinson's Disease, Auditory Cues, Gait

Citation. Matsumoto L, Magalhães G, Antunes GL, Torriani-Pasin C. Effect of rhythmic auditory cue on gait in patients with Parkinson's Disease
Trabalho realizado na Universidade Paulista de Santos, Santos-SP, Brasil.

1.Fisioterapeuta, bolsista de Iniciação Científica pela Universidade Paulista, Santos-SP, Brasil.

2.Fisioterapeuta, Especialista em Fisioterapia Neurológica, Supervisora de Estágio na Universidade Paulista, Santos-SP, Brasil.

3.Fisioterapeuta, Mestre, Professora adjunta do departamento de Fisioterapia, Universidade Paulista, Santos-SP, Brasil.

4.Fisioterapeuta, Doutora, Professora na Escola de Educação Física e Esporte,

Universidade de São Paulo, São Paulo-SP, Brasil.
Endereço para correspondência: Gisele Ladik Antunes Avenida Almirante da Gama, 141 apto 11, Ponta da Praia CEP 11030-401, Santos-SP, Brasil e-mail: giseleladik@hotmail.com 


\section{INTRODUÇÃO}

A Doença de Parkinson (DP) é a segunda mais comum desordem neurodegenerativa. Afeta cerca de 6 milhóes de pessoas ao redor do mundo e pelo menos 200 mil no Brasil ${ }^{1,2}$. Essa doença neurológica progressiva e crônica do sistema nervoso é caracterizada pela perda dos neurônios dopaminérgicos da parte compacta da substância negra, situada nos gânglios da base. A integração das informações sensitivas nos centros superiores necessários para a realização dos movimentos é comprometida, pois os núcleos de resposta dos gânglios da base (globo pálido e substância negra) sofrem forte inibição, dificultando a realização de movimentos sequenciais e da manutenção automática de seu ritmo e escala adequados ${ }^{3}$.

O quadro clínico característico compóe-se de distúrbios do movimento caracterizadas como sinais/sintomas da DP: hipocinesia, acinesia, tremor de repouso, rigidez muscular, bradicinesia e instabilidade postural ${ }^{1}$. Distúrbios da marcha estão entre os problemas mais preocupantes, podendo apresentar: diminuição no comprimento e na velocidade da passada, aumento na duração da fase de duplo apoio e pela redução na amplitude de movimento das articulaçôes do quadril, joelho e tornozelo, provocando marcha caracteristicamente lenta e $\operatorname{arrastada~}^{3-5}$. A cadência não é modificada em pacientes com DP atribuindo a hipocinesia à incapacidade de gerar grandes passos ${ }^{6}$; enquanto que outros estudos apontam aumento da cadência como um mecanismo de adaptação da redução do comprimento do passo ${ }^{7}$.

Os movimentos de tronco e da pelve também sofrem redução, resultando numa diminuição da oscilação recíproca dos braços. Ocorre anteriorização da cabeça e do tronco deslocando o centro de gravidade para adiante, podendo resultar em padrão de marcha festinada ${ }^{8}$.

Com a progressão da doença ocorre a piora das alteraçôes da marcha (tais como o congelamento de marcha e a rigidez) e o bloqueio motor, associadas às complicaçôes motoras (flutuaçôes e discinesias) que ocorrem em função do uso da levodopaterapia4. O bloqueio motor é classificado como episódico e definido como dificuldade de iniciar movimentos sequenciais, comprometendo o início da marcha ${ }^{2,9,10}$.

A natureza progressiva dos distúrbios da marcha pode conduzir a uma eventual perda de mobilidade, o aumento da incidência de quedas, perda da independência e redução da qualidade de vida ${ }^{4,6}$. Com três anos de diagnóstico 85\% dos indivíduos com DP provavelmente desenvolvem problemas na marcha ${ }^{4}$. Consequentemente, os tratamentos têm sido direcionados para que os pacientes mantenham a capacidade de caminhar independentemente.

Estudos têm demonstrado a eficácia do uso de estratégias de atenção na melhora dos parâmetros de marcha ${ }^{2,3,10,11}$. Quando um movimento é internamente gerado, existe um aumento relativo da atividade dos núcleos da base medial e das vias motoras complementares. Este circuito tem se mostrado hipoativo na DP. Com as pistas externas, as áreas parietal lateral, tálamo e as áreas pré-motoras são relativamente mais envolvidas ${ }^{3}$. Desse modo, ao direcionar a atenção para estímulos externos, pode-se aumentar a ativação muscular e a excitabilidade dos neurônios motores através da via retículo-espinal, desviando dos circuitos dos núcleos da base com déficits de dopamina ${ }^{12}$. O uso de Estímulos Acústicos Rítmicos (EAR) tais como metrônomo, batida musical ou ritmo de palmas têm sido implementado como estratégia na reabilitação de distúrbios da marcha ${ }^{13,14}$. Os EAR podem melhorar a marcha através da criação de uma união estável entre passos e a batida ${ }^{15}$. Na maioria dos estudos, os participantes são instruídos a sincronizar cada toque do pé no solo com o toque de um metrônomo. Um estudo realizado com idosos saudáveis evidenciou que ritmos mais próximos à frequência preferida resultaram em um acoplamento mais ideal entre a marcha e o ritmo apresentado $^{15}$. No entanto, os efeitos de pistas externas rítmicas nos parâmetros específicos da marcha em pessoas com DP ainda não são claros e merecem investigação mais aprofundada. Portanto, o objetivo do presente estudo foi analisar o efeito do estímulo acústico rítmico sobre os padrōes de marcha a fim de verificar quais parâmetros sofrem maior alteração no paciente com DP. Os resultados deste estudo podem contribuir para o aprimoramento da prática da fisioterapia, por meio da compreensão da dinâmica da marcha em resposta ao EAR.

\section{MÉTODO}

\section{Amostra}

A amostra foi composta por 21 indivíduos com 
idade média de $68,9 \pm 11,6$, sendo 15 homens e 6 mulheres com diagnóstico de DP idiopática. O tempo médio de diagnóstico foi de 6,8 anos. Os indivíduos foram recrutados de duas instituiçóes sendo uma clínica escola e uma Seção de recuperação e fisioterapia. O comprometimento e o estágio de evolução da doença foram avaliados através de uma entrevista inicial, da Unified Parkinson's Disease Rating Scale (UPDRS) e a escala de Hoehn \& Yahr modificada.

As condições cognitivas dos participantes foram mensuradas por meio do Mini Exame do Estado Mental (MEEM) validado para a população brasileira. Foram selecionados 21 pacientes, sendo utilizados os seguintes dos critérios de inclusão: (1) classificados entre estágios 1 e 3 da escala de Hoehn \& Yahr, (2) marcha independente (sem utilização de bengala ou andador) por pelo menos 50 metros, (3) ausência de comprometimento auditivo e vestibular e de doenças osteomusculares que os impediam de realizar a tarefa, (4) pontuação mínima no MEEM: analfabetos $=18$ pontos, com instrução escolar $=24 / 2516$

Este estudo foi aprovado pelo Comitê de Ética em Pesquisa da Universidade (protocolo número 169098131900055-12) e todos os participantes, após receberem todas as explicaçóes pertinentes ao estudo, assinaram o termo de consentimento livre e esclarecido.

\section{Procedimento}

Os participantes selecionados realizaram o Teste de caminhada dos 10 metros em dois momentos: um com marcha livre e outro com o EAR. O estímulo acústico foi fornecido pelo Software Metrônomo Plus e para análise espacial e temporal foram utilizados um cronômetro digital e canetas hidrocor, fixadas verticalmente com fita adesiva na parte posterior dos calçados dos pacientes (Figura 1), de modo que, ao andar, a ponta da caneta atingia o solo, marcando uma passadeira de papel pardo de 12 metros de comprimento e 60 centímetros de largura. Uma fita vermelha marcava o início e o final do percurso de 10 metros ${ }^{17}$.

Antes do início do teste, todos os indivíduos caminharam previamente (em passos habituais) sobre a passadeira para familiarizar-se com o teste. Nesse momento, foi definida a frequência de passos de cada indivíduo, ou seja, sua frequência preferida. Esta variável foi utilizada para determinar a frequência do EAR, personalizada como $10 \%$ acima da preferida para cada indivíduo. Para o início do teste, foi dado o sinal "pode ir", sendo que o cronômetro iniciou a contagem somente após a aceleração do paciente.

Por convenção, os indivíduos estavam calçados com sapatos fechados e de plataforma baixa. Os indivíduos caminharam em velocidade habitual. Após isso, repetiram o trajeto com o auxílio do estímulo acústico rítmico. Nas tentativas com EAR os participantes foram instruídos a sincronizar cada toque do pé no chão com cada toque sonoro do metrônomo. Após a preparação do participante e, antes de iniciar a coleta de dados, os participantes realizaram uma prática, para a compreensão da execução do teste.

\section{Avaliação}

Para avaliação cinemática quantitativa da marcha foram calculados a velocidade, a cadência e o comprimento do passo. Para análise de comprimento dos passos, foram descontados os primeiros três e os últimos três passos do percurso de 10 metros, que corresponde a aceleração e desaceleraçáo, utilizados os passos e passadas centrais para serem medidos. A seguir, foram medidas as distâncias entre os pontos marcados, com uma régua de $60 \mathrm{~cm}$ e um esquadro.

A cadência foi calculada dividindo-se o número total de passos pelo tempo em segundos, gastos no per-

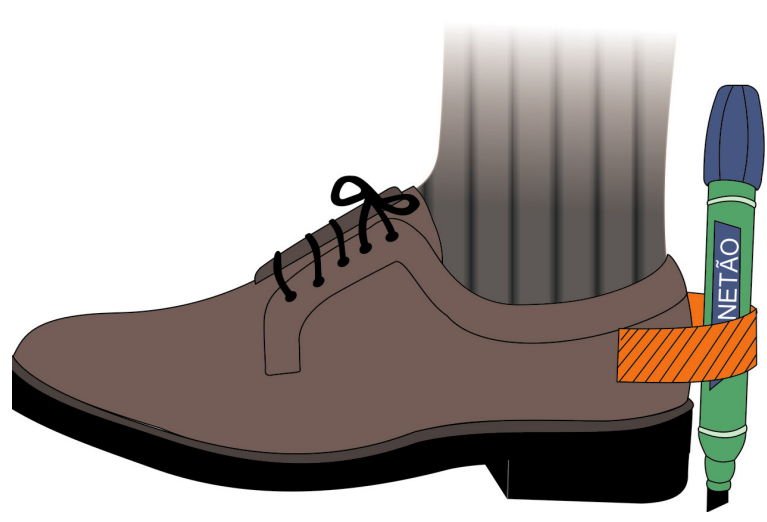

Figura 1. Disposição da caneta de demarcação para aferição do comprimento do passo. 
curso e multiplicado por 60 [C=P/T x 60]. A velocidade foi calculada dividindo-se a distância total pelo tempo gasto em segundos para vencer o percurso e multiplicado por $60[\mathrm{~V}=\mathrm{D} / \mathrm{T} \times 60]$.

\section{Análise Estatística}

Os dados foram analisados, usando o programa Excel para Mac (versão 2010). Os parâmetros de marcha (cadência, comprimento de passo e velocidade) foram comparados através do coeficiente de variação [CV $=100 \mathrm{x}$ desvio padrão/média] e para análise estatística foi utilizado o Teste t pareado para variáveis dependentes, considerando-se o nível de significância de 5\% para todas as análises.

\section{RESULTADOS}

Os dados relativos às variáveis demográficas e clínicos dos 21 participantes incluídos neste estudo foram apresentados na Tabela 1 .

\section{Efeito do EAR na marcha}

Em relaçáo ao efeito do EAR na velocidade de marcha observaram-se valores maiores para a condição com estímulo auditivo em comparação com a marcha livre, respectivamente $51,6 \pm 15,5$ e $45 \pm 13,6$ ( $\mathrm{p}<0,001$; Figura 2). O coeficiente de variação (CV) sobre a velocidade de marcha entre as duas condições resultou numa variação de 9,64\%.

O efeito da EAR na cadência de marcha mostrou valores maiores na presença do EAR comparado à marcha livre $112,0 \pm 15,3$ e 103,9 $\pm 15,2(\mathrm{p}<0,001$; Figura 2),

Tabela 1. Caracteristica da amostra.

\begin{tabular}{lccc}
\hline & $\begin{array}{c}\text { Idade } \\
\text { (anos) }\end{array}$ & Hoehn \& Yahr* & UPDRS** \\
\hline $\begin{array}{l}\text { Homens } \\
(\mathrm{n}=15)\end{array}$ & $66,9 \pm 11,9$ & 2,4 & $37,4 \pm 15,6$ \\
$\begin{array}{l}\text { Mulheres } \\
(\mathrm{n}=6)\end{array}$ & $73,7 \pm 10,3$ & 2,0 & $25,8 \pm 11,5$ \\
$\begin{array}{l}\text { Total } \\
(\mathrm{n}=21)\end{array}$ & $69,9 \pm 11,6$ & 2,3 & $34,1 \pm 15,3$ \\
\hline
\end{tabular}

*média do grupo; ** Unified Parkinson's Disease Rating Scale. resultando numa variação de 5,3\%.

Quanto ao comprimento do passo, apesar de apresentar uma variação menor 4,36\%, essa variável também sofreu um aumento na marcha com o uso do metrônomo $(50,7 \pm 14,4)$ em contraste com a marcha livre $(47,6$ $\pm 13,2$; $\mathrm{p}=0,015$; Figura 2).

\section{DISCUSSÂO}

Esse estudo avaliou os efeitos do estímulo acústico rítmico na marcha de pacientes com DP. Corroborando com estudos anteriores ${ }^{13,14,18}$, houve uma acentuada diferença na resposta aos EAR, que alterou significantemente os parâmetros de marcha nos indivíduos com DP, em especial a velocidade.

Observou-se aumento da velocidade com EAR e essa dica externa auditiva pode atuar como um alerta constante e marca-passo, reduzindo o custo atencional para a realização da marcha. Esse achado também foi observado em ambiente domiciliar, de modo que o uso de EAR nesse contexto e em ritmo igual à frequência preferida evidenciou melhoras na velocidade e no comprimento médio do passo ${ }^{19}$. Por outro lado, utilizando um ritmo de $25 \%$ acima da marcha preferida e também houve diferenças na velocidade e na cadência, aumentando em $13 \%$ e $9 \%$ respectivamente, porém nenhum efeito sobre o comprimento do passo ${ }^{18}$.

Nossos achados assemelham-se a outro estudo em que foi utilizado ritmo $10 \%$ abaixo da frequência preferida encontraram aumento da velocidade da marcha e comprimento do passo, no entanto uma reduçâo da cadência ${ }^{20}$. Não foram observadas diferenças tanto no EAR calculado em $10 \%$ acima da frequência preferida quanto em $10 \%$ abaixo $^{21}$. Desse modo, pode ser que a frequência da EAR não seja o fator fundamental na estimulação, pois somente sua presença como pista externa para marcação da marcha já evidencia melhora nos parâmetros da mesma.

Como explicações neurofisiológicas para os efeitos encontrados com o uso de EAR tem-se que os padróes sonoros rítmicos podem aumentar a excitabilidade dos neurônios motores espinais, através da via retículo espinal, reduzindo a quantidade de tempo necessário para os músculos responderem a um determinado comando motor $^{18,22}$. Nesse contexto, estudos com registros eletro- 
Figura 2. Média dos valores de velocidade, cadência e comprimento do passo para condiçōes de marcha livre e marcha com Estímulo Acústico Rítmico (EAR).
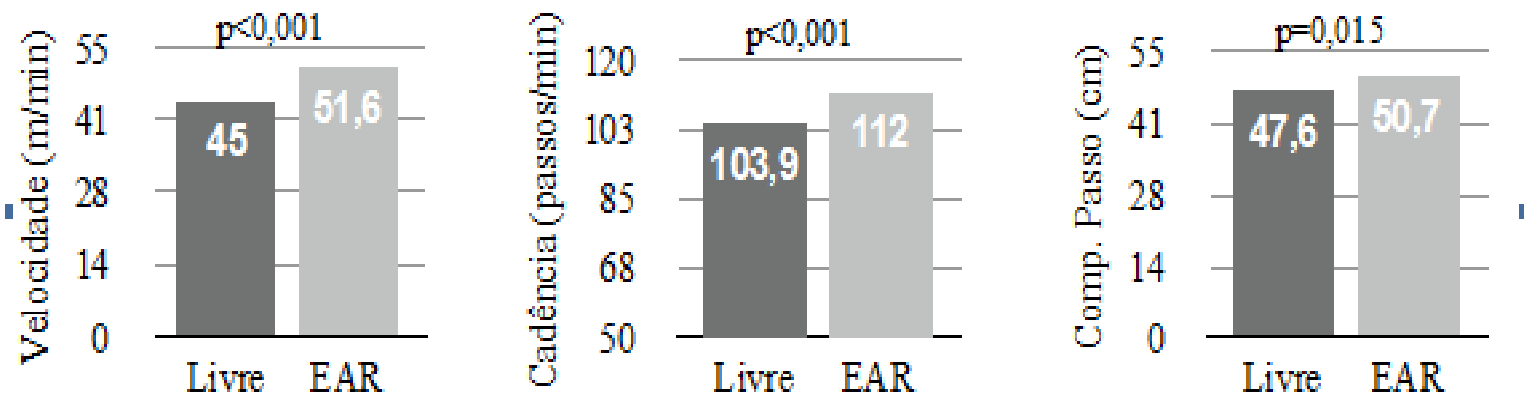

miográficos no músculo gastrocnêmio de indivíduos com DP enquanto caminhavam durante a estimulação auditiva com ritmo fixado ilustram que os EAR podem sincronizar os movimentos com a estimulação promovendo maior consistência no padrão de recrutamento de unidades motoras ${ }^{22}$.

Há, ainda, outras especulações relativas aos afeitos fisiológicos da estimulação auditiva. Foram reladas mudanças na captação de glicose em várias áreas do cérebro envolvidas no processamento sensitivo-motor após um programa de reabilitação usando EAR, fornecendo novas informaçôes sobre a base neural da estimulação auditiva na $\mathrm{DP}^{12}$.

Os resultados dessa pesquisa apontam para a possibilidade de incluir o uso de EAR como uma ferramenta para o tratamento da marcha de pacientes com DP.

O presente estudo apresenta algumas limitaçóes acerca da metodologia e desenho experimental utilizados, tais como: a ausência de um grupo controle, a avaliaçáo dos efeitos imediatos (e não tardios) e o tamanho amostral pequeno. Nesse contexto, o delineamento de um estudo que investigue os efeitos do uso da EAR em longo prazo, com uma amostra maior e na presença de um grupo controle são fundamentais para a certificação dos dados encontrados.

\section{CONCLUSÃO}

Os resultados do presente estudo apontaram que o uso de estímulo acústico rítmico, em 10\% acima da frequência preferida, modifica os parâmetros da marcha, com maior alteração na velocidade em comparação à cadência e comprimento de passo em indivíduos com doença de Parkinson.

\section{REFERÊNCIAS}

1.Scalzo PL, Flores CR, Marques JR, Robini SCO, Teixeira AL. Impact of changes in balance and walking capacity on the quality of life in patients with Parkinson's disease. Arq Neuropsiquiatr 2012;70:119-24. http://dx.doi. org/10.1590/S0004-282X2012000200009

2.Ford MP, Malone LA, Nyikos I, Yelisetty R, Bickel CS. Gait training with progressive external auditory cueing in persons with Parkinson's disease. Arch Phys Med Rehabil 2010;91:1255-61. http://dx.doi.org/10.1016/j. apmr.2010.04.012

3.Nieuwboer A, Rochester L, Jones D. Cueing gait and gait-related mobility in patients with Parkinson's disease. Top Ger Rehab 2008;24:151-65.

4.Kelly VE, Eusterbrock AJ, Shumway-Cook A. A Review of dual-task walking deficits in people with Parkinson's disease: motor and cognitive contributions, mechanisms, and clinical implications. Parkinsons Dis 2012;2012:918719. doi: 10.1155/2012/918719. http://dx.doi.org/10.1155/2012/918719

5.Mcintosh GC, Brown SH, Rice RR, Thaut MH. Rhythmic auditory-motor facilitation of gait patterns in patients with Parkinson's disease. J Neurol Neurosur Psychiatr 1997;62:22-6.

6.Morris ME, Iansek R, Matyas TA, Summers JJ. Ability to modulate walking cadence remains intact in Parkinson's disease. J Neurol Neurosur Psychiatr 1994;57:1532-4.

7.Azulay JP, Mesure S, Blin O. Influence of visual cues on gait in Parkinson's disease: Contribution to attention or sensory dependence. J Neurol Sci 2006;248:192-5. http://dx.doi.org/10.1016/j.jns.2006.05.008

8.Morris ME. Movement disorders in people with Parkinson disease: A model for physical therapy. J Am Phys Ther Assoc 2000;80:578-97.

9.Morris ME, Martin CL, Schenkman ML. Striding out with Parkinson disease: evidence-based physical therapy for gait disorders. J Am Phys Ther Assoc 2010;90:280-8. http://dx.doi.org/10.2522/ptj.20090091

10.Seco-Calvo J, Gago-Fernández I, Cano-De-La-Cuerda R, Fernández-De-Las-Penas C. Efectividad de los estímulos sensoriales sobre los trastornos de la marcha en pacientes con enfermedad de Parkinson. Estudio piloto. Rev Fisioter 2012;34:4-10. http://dx.doi.org/10.1016/j.ft.2011.07.009 
11.Baker K, Rochester L, Nieuwboer A. The immediate effect of attentional, auditory, and a combined cue strategy on gait during single and dual tasks in Parkinson's Disease. Arc Phys Med Rehab 2007;88:1593-600. http://dx.doi. org/10.1016/j.apmr.2007.07.026

12.Del Olmo MF, Arias P, Furio MC, Pozo MA, Cudeiro J. Evaluation of the effect of training using auditory stimulation on rhythmic movement in Parkinsonian patients - a combined motor and study. Parkinsonism Relat Disord 2006;12:155-64. http://dx.doi.org/10.1016/j.parkreldis.2005.11.002

13.Luessi F, Mueller LK, Breimhorst M, Vogt T. Influence of visual cues on gait in Parkinson's disease during tredmill walking at multiples velocities. J Neurol Sci 2012;314:78-82. http://dx.doi.org/10.1016/j.jns.2011.10.027

14.Rochester L, Baker K, Hetherington V, Jones D, Willems AM, Kwakkel G, et al. Evidence for motor learning in Parkinson's disease: Acquisition, automaticity and retention of cued gait performance after training with external rhythmical cues. Brain Res 2010;1319:103-11. http://dx.doi.org/10.1016/j. brainres.2010.01.001

15.Roerdink M, Bank P, Beek P. Walking to the beat of different drums: Practical implications for the use of acoustic rhythms in gait rehabilitation. Gait Post 2011;33:690-4. http://dx.doi.org/10.1016/j.gaitpost.2011.03.001

16.Lourenço RA, Veras RP. Mini-Exame do Estado Mental: Características psicométricas em idosos ambulatoriais. Rev Saude Publ 2006;40:712-9. http:// dx.doi.org/10.1590/S0034-89102006000500023
17.Henriques GRP, Ribeiro ASB, Corrêa AL, Sanglard RCF, Pereira JS. A interferência da redução progressiva nas amplitudes da articulação coxo-femural na velocidade da marcha. Fit Perfor J 2003;2:183-9.

18.Suteerawattananon M, Morris GS, Etnyre BR, Jankovic J, Protas EJ. Effects of visual and auditory cues on gait in individuals with Parkinson's disease. J Neurol Sci 2004;219:63-9. http://dx.doi.org/10.1016/j.jns.2003.12.007

19.Rochester L, Hetherington V, Jones D, Nieuwboer A, Willems A, Kwakkel G, Wegen EV. The effect of external rhythmic cues (auditory and visual) on walking during a functional task in homes of people with Parkinson's disease. Arch Phys Med Rehabil 2005;86:999-1006. http://dx.doi.org/10.1016/j. apmr.2004.10.040

20.Baker K, Rochester L, Nieuwboer A. The effect of cues on gait variability - Reducing the attentional cost of walking in people with Parkinson's disease. Parkinsonism Relat Disord 2008;14:314-20. http://dx.doi.org/10.1016/j.parkreldis.2007.09.008

21.Lohnes CA, Earhart GM. The impact of attentional, auditory, and combined cues on walking during single and cognitive dual tasks in Parkinson disease. Gait Post 2011;33:478-83. http://dx.doi.org/10.1016/j.gaitpost.2010.12.029 22.Del Olmo MF, Cudeiro J. Simple procedure using auditory stimuli to improve movement in Parkinson's disease: A Pilot Study. Neurol Clin Neurophysiol 2003;2:1-7. 